\title{
RBD-specific antibody responses after two doses of BBIBP-CorV (Sinopharm, Beijing CNBG) vaccine
}

Tamás Ferenci ${ }^{1,2}$ and Balázs Sarkadi ${ }^{3,4^{*}}$

\begin{abstract}
Background Limited information is available on the effectiveness of the BBIBP-CorV (Sinopharm, Beijing CNBG) vaccine, especially in the elderly, despite the fact that it is approved in more than 50 countries.

Methods RBD-specific antibody titres, as a rapidly available and highly predictive surrogate marker, were measured after two doses of the BBIBP-CorV vaccine in 450 subjects. Results were analyzed in a multivariable model accounting for age, sex and time since the administration of the second dose of the vaccine.

Results Sex and time since the second dose had little association with the antibody titres. Age, however, was highly relevant: measurable antibody levels were present in about $90 \%$ of individuals below the age of 50 , but antibody production after BBIBP-CorV vaccination was strongly reduced with increasing age. A large number of elderly subjects, reaching $25 \%$ at 60 years, and up to $50 \%$ at ages over 80 , were found not to produce any protective antibody.

Conclusions RBD-specific antibody titre, as a correlate of protection for COVID-19 disease susceptibility, should help to evaluate the effectiveness of the BBIBP-CorV vaccine. Results suggest that proper measures should be undertaken to prevent a potential outbreak of COVID-19 in BBIBP-CorV vaccinated but eventually unprotected elderly individuals.
\end{abstract}

Keywords COVID-19, SARS-CoV-2, Vaccine, BBIBP-CorV, Antibody response

\section{Background}

The use of the BBIBP-CorV (Sinopharm, Beijing CNBG) vaccine in Hungary was authorized by the Hungarian national drug and food evaluation authority, based on a governmental decree in January [1]. At the time of this authorization there were no publicly available Phase 3

\footnotetext{
${ }^{*}$ Correspondence:

Balázs Sarkadi

sarkadi.balazs@ttk.hu

1 Physiological Controls Research Center, Óbuda University, Bécsi út 96/b, Budapest 1034, Hungary

2 Department of Statistics, Corvinus University of Budapest, Fővám tér 8, Budapest 1093, Hungary

${ }^{3}$ Institute of Enzymology, ELKH Research Centre for Natural Sciences,

Magyar Tudósok krt. 2, Budapest 1117, Hungary

${ }^{4}$ Department of Biophysics and Radiation Biology, Semmelweis

University, Túzoltó u. 37-47, Budapest 1094, Hungary
}

evaluation data, and unpublished data partially released by the Hungarian authorities suggested that limited information was available about the use of the Sinopharm vaccine, especially in elderly people: these interim data reported that $99.3 \%$ of the subjects in Phase 3 was below 60 [2]. As of 27 June 2021, 1.05 million people received the first dose with this vaccine in Hungary, and 1 million received the second dose too [3]. Of these, more than half (54.1\% from those receiving the first dose) were above the age of 60 [3]. Since no other EU countries authorized the use of Sinopharm vaccine, it is especially important to follow the potential protective effect of this vaccination in an EU member country, focusing on elderly people.

Correlates of protection [4] for SARS-CoV-2 are not yet firmly established [5], but a growing body of evidence suggests that neutralizing antibody levels are 
highly predictive for protection against symptomatic disease [6-13]. While very limited empirical evidence exists specifically about the Sinopharm vaccine in this respect, traditional immunology knowledge and experience with prior vaccines suggest that the technology of the Sinopharm vaccine (whole inactivated virion with alum adjuvant) results in a Th2-skewed immune response $[14,15]$. This also suggests that in case of the Sinopharm vaccine, antibody response may be a good correlate, which is further reinforced by the fact that in case of a technologically very similar SARS-CoV-2 vaccine, CoronaVac, the Phase 2 study failed to find clear evidence of cellular response using ELISpot [16]. (Such study was unfortunately not carried out during the Phase 2 of the Sinopharm vaccine [17].)

On May 24, 2021, a report on the Phase 3 trial of the Sinopharm vaccine, showing positive efficacy and safety data, was finally published [18]. However, the paper revealed that $84.4 \%$ of the trial participants were male, $98.4 \%$ were aged less than 60 years and $100 \%$ were healthy (as it was an inclusion criterion, with "healthy" being investigator-judged with no precise definition). This is in stark contrast with the trials of vaccines that are authorized in other EU countries [19], for instance, in the Phase 3 trial of the BNT162b2 vaccine (Comirnaty, Pfizer/BioNTech) $42.2 \%$ of the subjects was above 55 years of age [20] and $20.3 \%$ had one or more underlying disease [21]. As a result, there is very little direct evidence available about the efficacy and safety of Sinopharm vaccination among women, in elderly, and those with underlying diseases, thus a study in these regards is highly important, especially as these are also largely the risk groups for SARS-CoV-2 infection, and are known to be associated with lower vaccine effectiveness at the same time. In particular, lower vaccines effectiveness among the elderly is well-documented for several vaccines [22-24], and has already been described specifically for SARS-CoV-2 too [25]. Several groups with underlying disease, such as organ transplant recipients are also at higher risk of vaccine failure [26-28].

In addition, news reports from the United Arab Emirates, Serbia and other countries using mostly the Sinopharm vaccine indicated limited protection against SARS-CoV-2 virus infection in elderly people after vaccination with Sinopharm. Anecdotal cases from Hungary also pointed to possible inefficient protection by even two doses of Sinopharm vaccine: several hundreds of such test results were informally reported in the press and social media.

Therefore, a proper analysis of virus neutralizing antibody measurements, or RBD-specific antibody measurements (which have been shown to correlate with neutralizing antibody measurements) should have a major importance in evaluating the potential efficacy of Sinopharm in elderly, thus helping to prevent a potential outbreak of COVID-19 in vaccinated but eventually unprotected individuals. This question is crucial as more than 50 countries approved the Sinopharm vaccine as of late June, 2021 [29] and it is on the World Health Organization's Emergency Use Listing [30].

\section{Methods}

Antibody measurements (initiated by the vaccinated individuals who sought an approved clinic for antibody testing) after at least 14 days of obtaining the second vaccine dose of the BBIBP-CorV (Sinopharm, Beijing CNBG) vaccine were collected from $>18$ years old subjects. Known prior SARS-CoV-2 or current infection were exclusion criteria, but subjects were otherwise unselected. Information on the age and sex of the subjects and the time past after the second dose were also collected. All subjects signed a full written informed consent allowing the later publishing and anonymous statistical analysis of the collected data. De-identified data published based on this consent were openly available to the public before the initiation of the study and were acquired to carry out this analysis. These data can be found-along with the full analysis script-at https:/github.com/ tamas-ferenci/SinopharmAntibodyResponse. All methods were carried out in accordance with relevant guidelines and regulations. The protocol for measurements was approved by the Hungarian health authorities.

After obtaining venous blood samples from the individuals, the RBD-specific antibody measurements were performed in an accredited laboratory using an FDA emergency use approved and internationally accredited method (ADVIA Centaur SARS-CoV-2 IgG, COV2G), shown to correlate with direct virus neutralizing tests [31]. For a comparison, we have also obtained anonymous RBD-specific antibody laboratory data for 45 individuals, vaccinated by two doses of the Pfizer/BioNTech RNA-based vaccine. RBD-specific antibody titres provided by the applied antibody assay are measured in the "Centaur index" $(\mathrm{Ci})$ arbitrary unit and are categorized according to the manufacturer as follows:

less than 1 Ci: no RBD-specific antibody present,

1-10 Ci: moderate RBD-specific antibody titre,

10-30 Ci: strong RBD-specific antibody titre,

30-150 Ci: outstandingly strong RBD-specific antibody titre.

The applicability of antibody titres, as correlating measures with the COVID-19 disease, has not been fully clarified in the relevant literature [32-36]. While rapid, lateral flow or other IgG-IgM test procedures may yield questionable results, the properly assayed antibody titres have 
been documented to correlate with disease susceptibility. This is especially true for accredited laboratory assays, as applied here, measuring antibody binding to the receptor binding domain (RBD) of the Spike virus protein, having a key role in the binding and cellular entry of the SARS$\mathrm{CoV}-2$ virus. This type of assay, that is RBD-binding antibody measurements, have been used in the Phase 1-2 studies of the RNA-based vaccines [37], and has been shown to correlate with direct virus neutralizing assays [31, 38], not available for larger scale studies. Quantification of neutralizing antibody levels is clearly essential for vaccine development and for assessing protective immunity both at the individual and community levels. In addition, RBD binding virus neutralizing antibodies are most promising therapeutic tools for preventing or curing the COVID-19 disease $[39,40]$.

To investigate the effects of age, sex and time after the second dose, a multivariable model was used in which these variables were entered as covariates, with the response being the titre. Age and time after the second dose were spline-expanded with thin plate regression splines to allow for a potentially non-linear effect [41]. Given the high number of zero titre measurements and the highly skewed nature of the non-zero measurements, a hurdle-lognormal model was used, with the same formula for the hurdle. As an alternative analysis, standard logistic regression was applied with the titre dichotomized at $1 \mathrm{Ci}$ cutoff (no protective antibody), with the same covariates and similar spline expansion. In case of the Pfizer/BioNTech vaccine, antibody titres were measured exactly 28 days after the second dose in all subjects, so time since the second dose was not included in these investigations, but the models were otherwise the same as for the Sinopharm data.

Statistical analysis was carried out under the R statistical environment version 4.1.0 [42] using package brms version 2.15.0 [43]. Models were estimated in a Bayesian approach with default weakly informative priors of brms applying the Markov Chain Monte Carlo (MCMC) method using 4 chains and 2000 iterations with 1000 warmup [43]. Convergence was assessed with the potential scale reduction factor (Gelman-Rubin statistic) Rhat. Detailed model diagnostics is presented in the Additional file 1.

Results are presented as predicted probabilities that the titre is below a certain threshold, which was calculated by obtaining 4000 posterior samples of the linear predictor and then the probability was manually calculated taking the hurdle into account. The sample size for the Sinopharm vaccinated subjects was large enough so that the estimates are sufficiently precise, as evidenced by the width of the credible interval.

\section{Results}

Within the period of April 1-June 21, 2021, 497 measurements were obtained from Sinopharm-vaccinated study participants. Of these, 6 had no information on the date of the second dose and 1 had missing data on age; of the remaining 490 measurements, 40 was carried out within

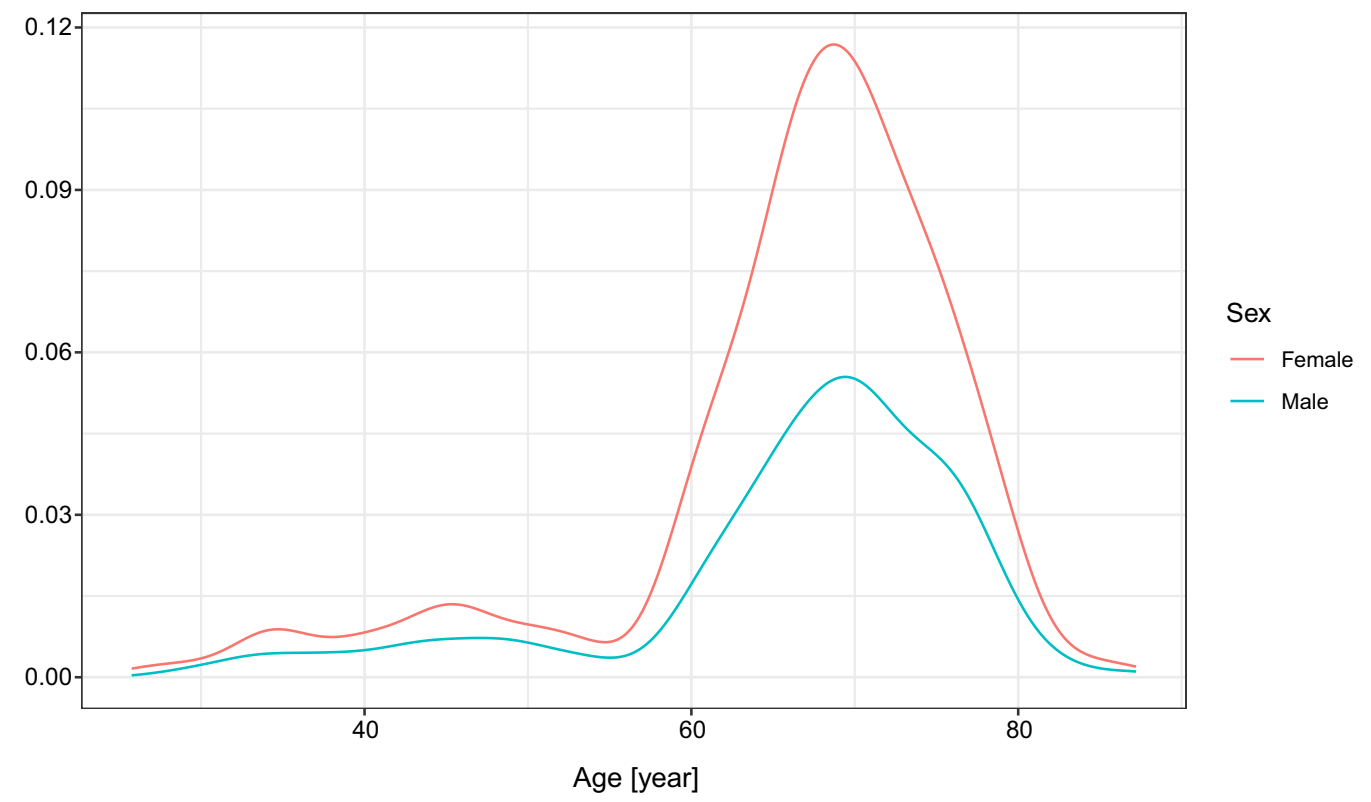

Fig. 1 Age distribution of individuals for RBD-specific antibody measurements after two doses of Sinopharm vaccine 
14 days of the second dose. Excluding these resulted in a final dataset with $n=450$ subjects that was analyzed.

Figure 1 shows the age distribution of the individuals, both male and female, participating in the antibody measurements. Since these were measurements initiated by private persons, mostly elderly individuals requested the antibody measurements, and the peaks of the curves are about the ages $65-75$ for both sexes. Still, data from numerous younger and older individuals are also included in the present analysis. Of the participants, 208 (46.2\%) were male.

The median time after the second dose was 23 days (lower quartile: 18 days, upper quartile 30 days).

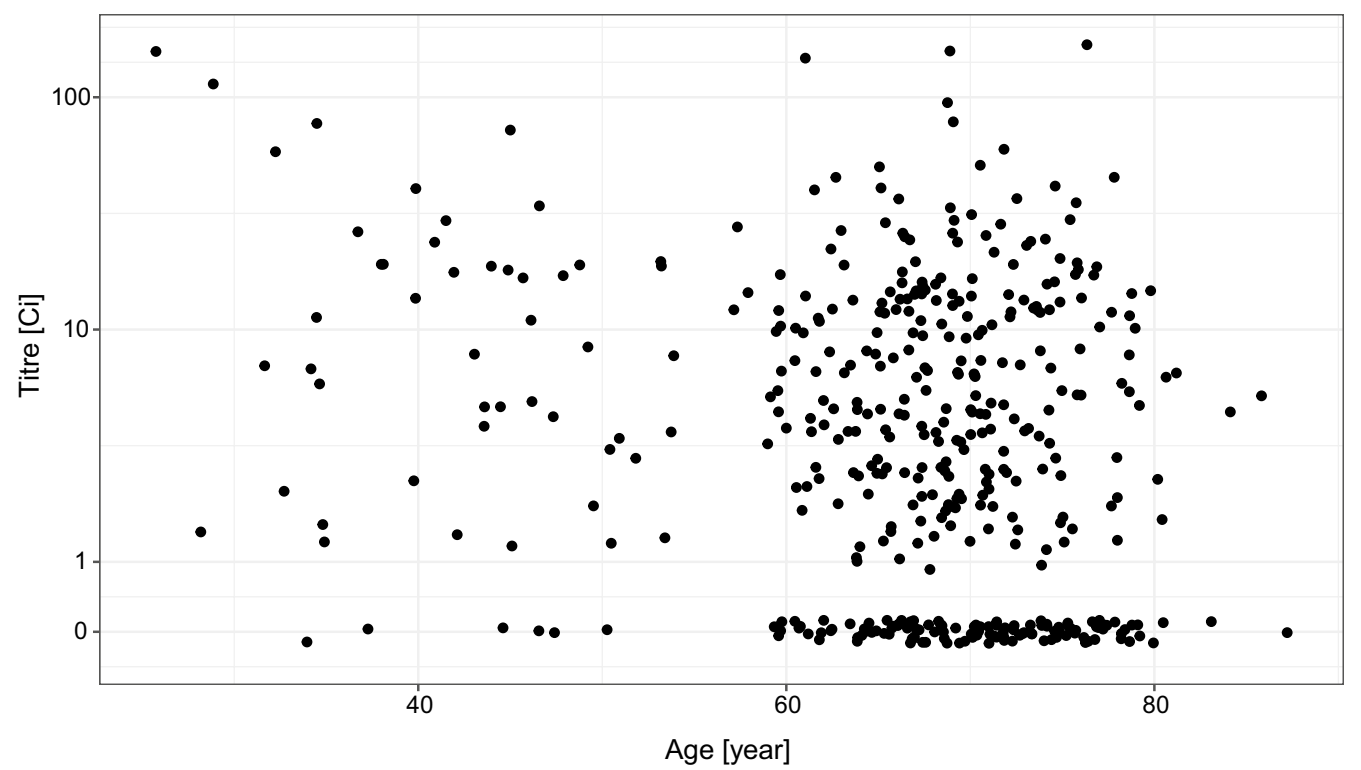

Fig. 2 Distribution of the virus RBD-specific titre according to the age of the subjects after two doses of the Sinopharm vaccine shown as jittered scatterplot. (Data are shown in pseudo-log scale.)

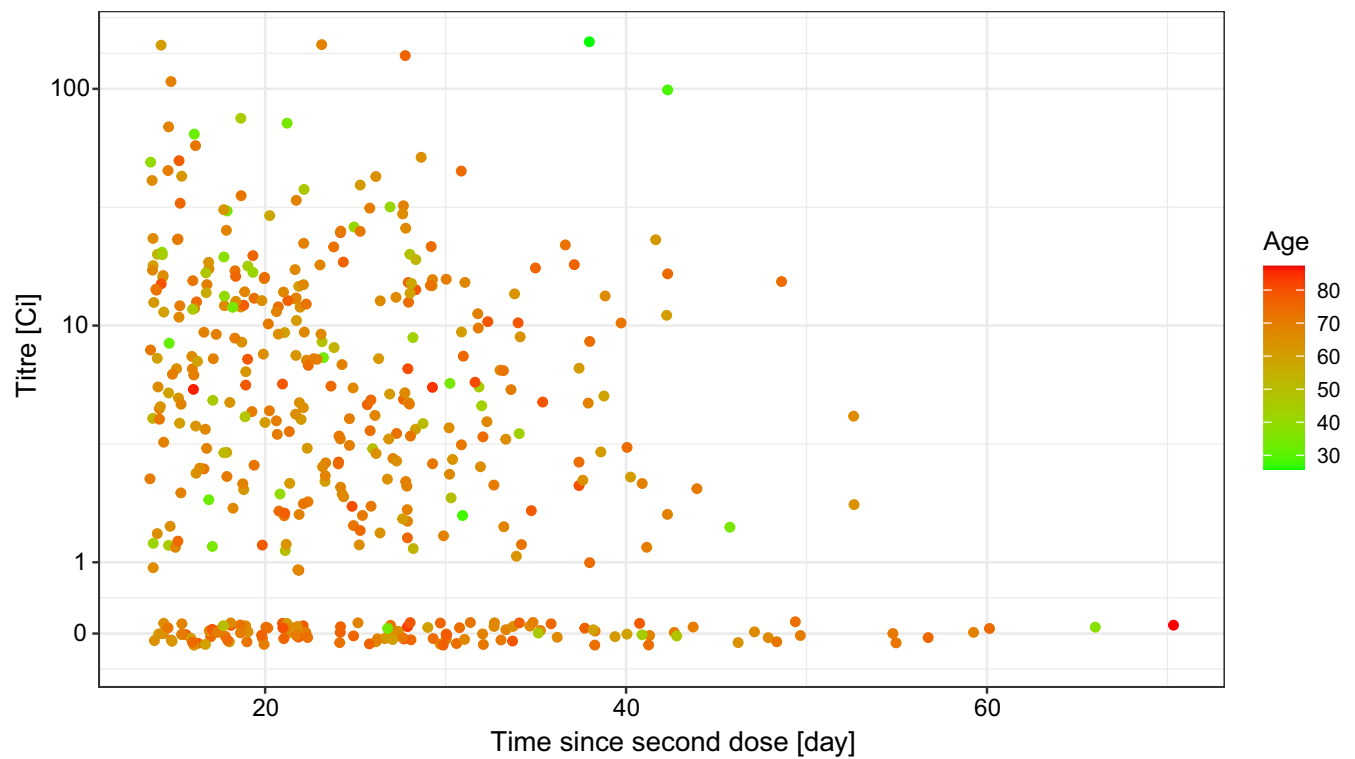

Fig. 3 Distribution of the RBD-specific antibody titre according to the time-period of the antibody measurement following the vaccination by two doses of the Sinopharm vaccine shown as jittered scatterplot. (Data are shown in pseudo-log scale.) 
As shown in Fig. 2, the overall antibody titre showed a decreasing tendency by the increasing age, moreover, a large number of subjects with no detectable antibody was present in the elderly population.

The distribution of the measured antibody titre showed no apparent univariate association either with the number of days since the second dose (Fig. 3), or with the sex of the subjects (Fig. 4). As far as the former is concerned, note that the range of the data-as Fig. 3 shows-was very narrow in terms of time since vaccination, thus realistically, no conclusion on the waning of vaccine effectiveness can be expected.

In the following, we performed a multivariable modelling to estimate the probability of having low RBDspecific antibody titre after obtaining two doses of the Sinopharm vaccine. Rhat was 1.00 for all parameters, indicating that the used algorithm converged, meaning that the results are reliable in this respect.

As shown in Fig. 5, the increasing age of the subjects shows a significant correlation with the lack of antibody production (titre below 1, no response), or low antibody titre (titre below 10, weak response). At about the age of 60 years, the estimated probability of the lack of antibody response was about $25 \%$, and this value increased up to about $50 \%$ at the age of 80 years. Regarding the expected low titre response, at the age of 60 years this reached $60-75 \%$, further increasing with increasing age.

The number of days between the vaccination and the antibody measurement had little negative effect on the antibody titre, while in the case of female subjects the estimated probability for the lack of antibody production or lower antibody titre was somewhat smaller.

Logistic regression analysis confirmed these findings as shown in the Additional file 1 (Figs. S1, S2).

We have data only for a relatively small number (45) of subjects vaccinated by the Pfizer/BioNTech vaccine (Fig. 6). Despite that, the model indicates a drastically lower probability of having low, and especially zero antibody titre in the vaccinated individuals (Fig. 7). The increasing age also had a negative effect on the antibody titre in the case of the Pfizer/BioNTech vaccine, but no subject was found in this group producing no antibody (that is, a titre below 1), the probability of which was estimated to be less than $10 \%$ even in the oldest age group.

\section{Discussion}

In this study we have analyzed the SARS-CoV-2 virus RBD-specific antibody titre, shown to correlate with neutralizing antibody titre, as a potential surrogate for disease protection, in subjects vaccinated with the Sinopharm inactivated virus vaccine.

Several studies investigated the immunogenicity of the Sinopharm vaccine. Feng et al. report a clinical trial on the immunogenicity of the vaccine [44], but no information is given on the proportion of elderly subjects, and the mean age was 38.8 years. Ariamanesha et al. investigated the Sinopharm vaccine among recipients with malignancy [45], with ample number of elderly subjects,

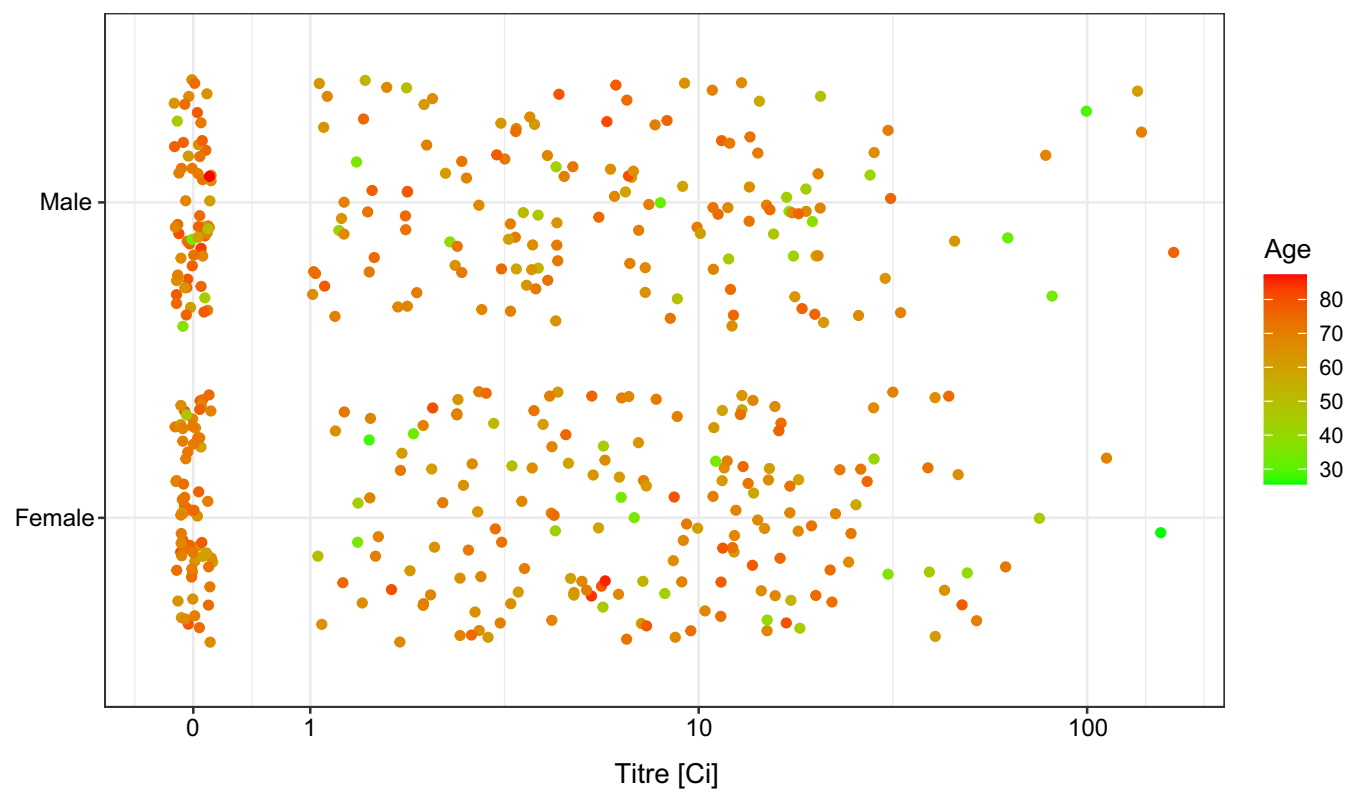

Fig. 4 Distribution of the RBD-specific antibody titre according to the sex of the subjects after two doses of the Sinopharm vaccine. (Data are shown in pseudo-log scale.) 


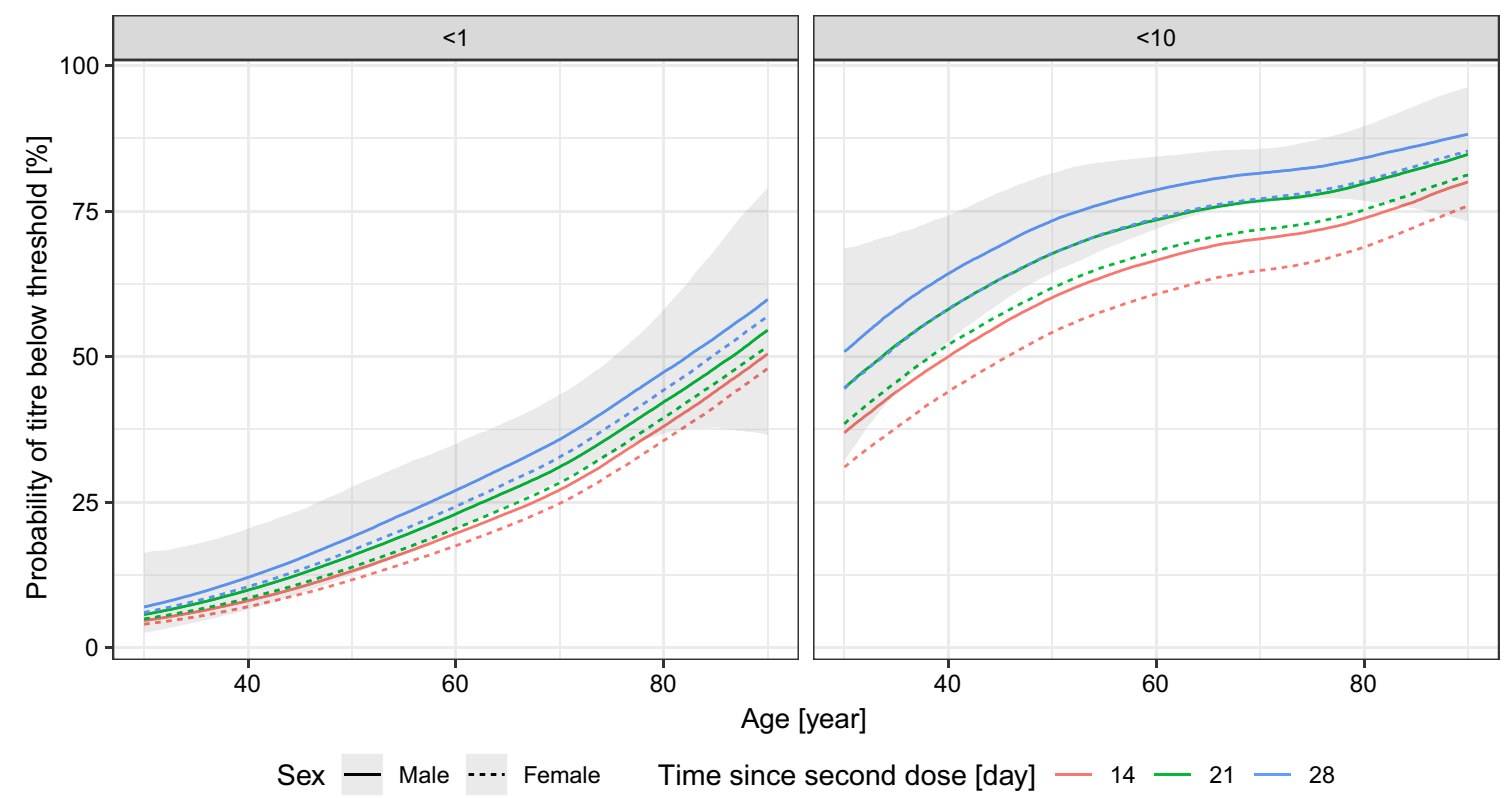

Fig. 5 Effects of the age and the sex of the subject, and the time-period between the vaccination and the measurement on the probability of the lack of RBD-specific antibody production (titre below 1, left panel) and of low antibody titre (titre below 10, right panel) after two doses of the Sinopharm vaccine. 90\% credible interval is shown for males, 28 days post second dose (credible intervals for the other curves are not shown for visual clarity, as they are very similar)

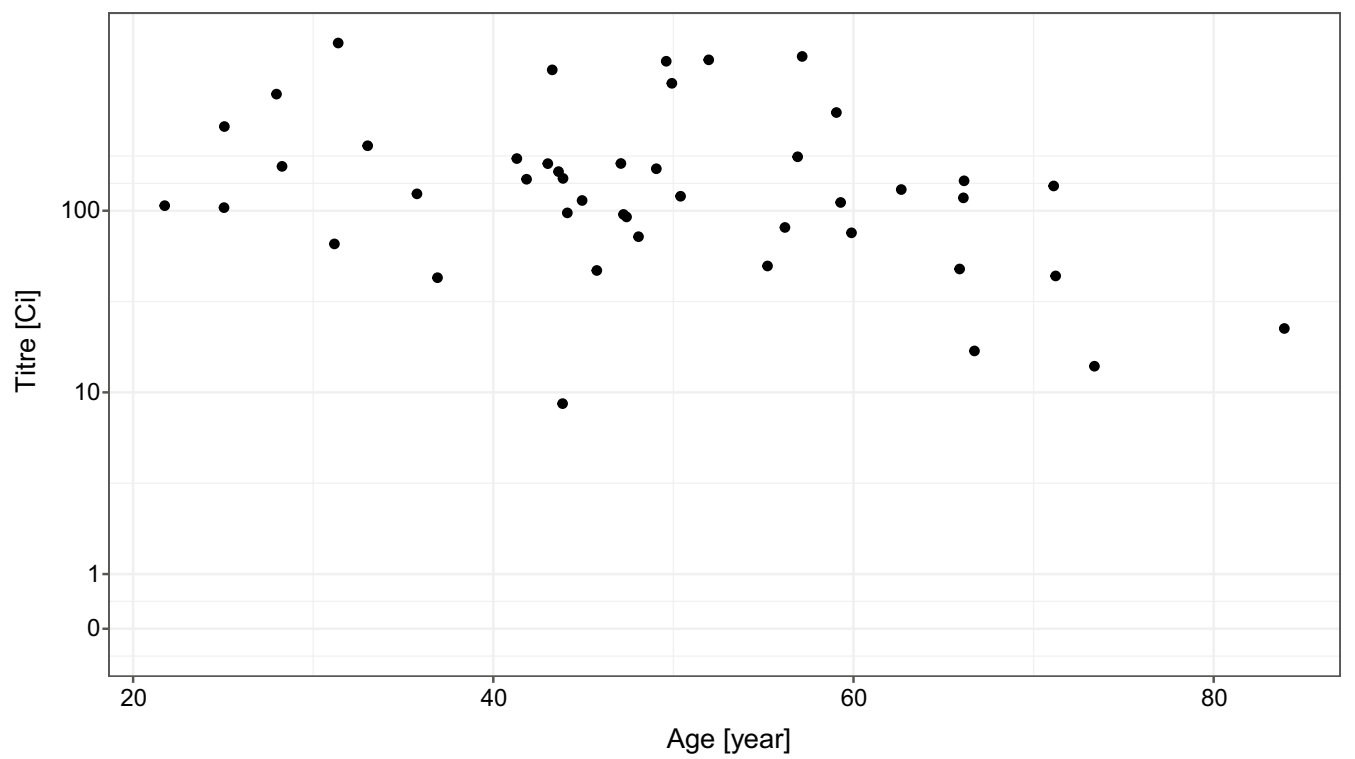

Fig. 6 Distribution of the virus RBD-specific titre according to the age of the subjects after two doses of the Pfizer/BioNTech vaccine shown as jittered scatterplot. (Data are shown in pseudo-log scale.)

and presented results according to age. These were similar to our findings, with only $75.3 \%$ being antibody positive above 60 years of age.

Alqassieh et al. [46] estimated a multivariable model which also showed drastically lower probability of positivity in those above 60 years of age (odds ratio less than
0.2 compared to those below 60). Fu performed an extensive analysis on a small number of patients [47] but did not report data by age, and all subject was below 60 . The antibody and $\mathrm{T}$ cell response study of Vályi-Nagy et al. was carried out in Hungary just as ours [48], but unfortunately included almost no elderly subjects. This study, 


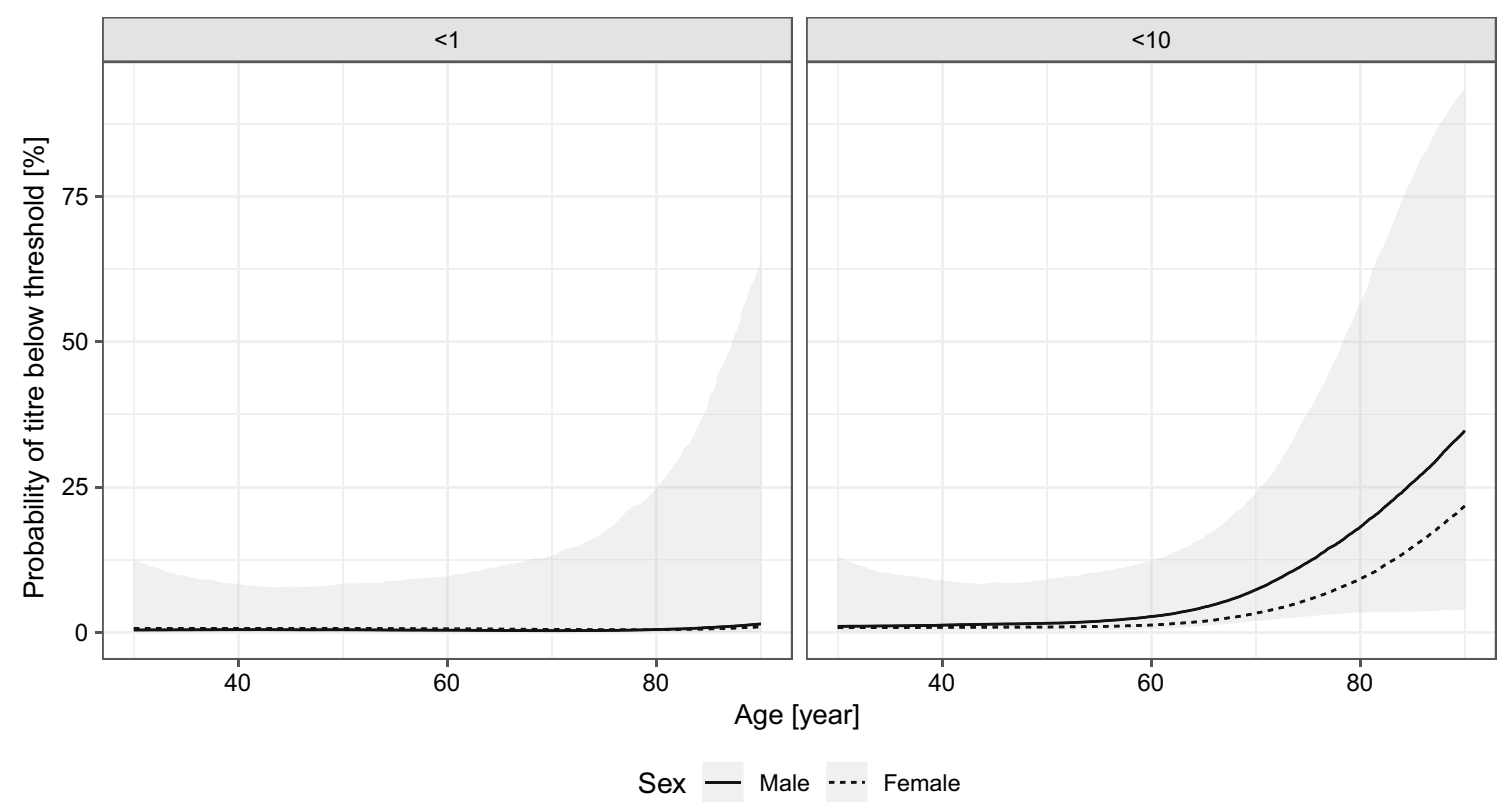

Fig. 7 Effects of the age and sex of the subject on the probability of the lack of RBD-specific antibody production (titre below 1, left panel) and of low antibody titre (titre below 10, right panel) after two doses of the Pfizer/BioNTech vaccine. $90 \%$ credible interval is shown for males, 28 days post second dose (credible intervals for the other curves are not shown for visual clarity, as they are very similar)

however, did find cellular response even with the Sinopharm vaccine. The immunogenicity study from Lijeskić et al. reporting data from Serbia [49] did not find lower antibody response in older subjects, however, the sample size was small.

Perhaps the most comparable to our research was that of Dashdorj et al. and that of Jeewandara et al. The former investigated the antibody responses in Mongolia [50] and found results similar to ours: titres were lower in patients above 60 years of age. In contrast to our work, however, that study made no attempt to investigate the impact of age as a continuous variable. The latter study was a comprehensive investigation carried out in Sri Lanka [51]. They also made no attempt to continuously model age, but dichotomization at 60 years revealed significantly lower titres in older subjects. The values were however much higher than in our study (93\% being seropositive even in those aged above 60) but measured only 14 days after the second dose. Of note, they did detect significant cellular response. A follow-up study from the same group [52], repeating the measurements at 3 months after the second dose, largely reconciles the findings with ours: they report substantial reduction in antibody response, and-coherently with our finding-especially in the elderly (with a specific antibody positivity in only $38.1 \%$ of those above 60).

Very few studies addressed the real-life effectiveness of the first two doses of the Sinopharm vaccine, especially in the elderly. Li et al. carried out a test-negative case control study, but its population included no subject above 60 years of age [53]. A large cohort study from Macchia et al. [54] reported data from Argentina, using only elderly subjects and providing detailed breakdown according to age, however, they did not present data according to vaccine type, and only $11.6 \%$ of the vaccinated population received the Sinopharm vaccine.

Of particular importance is the well-designed, largesample observational cohort study from Vokó et al. [55] which investigated, among others, the Sinopharm vaccine's real-life clinical effectiveness in Hungary between 22 January 2021 and 10 June 2021, and presented the results stratified according to fine-resolution age groups. This study is particularly interesting as it discusses the same vaccine in the same population and in the same time period, but uses a clinical outcome, thus it provides a unique opportunity to contrast antibody levels with clinical protection. Figure 8 shows the clinical vaccine effectiveness against infection from the Vokó et al. paper, overplotted with the probability of antibody positivity ( $>1 \mathrm{Ci}$ titre) from the present study. Remarkably, the two curves almost coincide. Note however, that we were not able to match the time since vaccination (which is not investigated in the Vokó et al. paper, and the present study, as already noted, provides little data on it).

Under the current pandemic circumstances, the reliable estimation of a direct relationship between the risk 


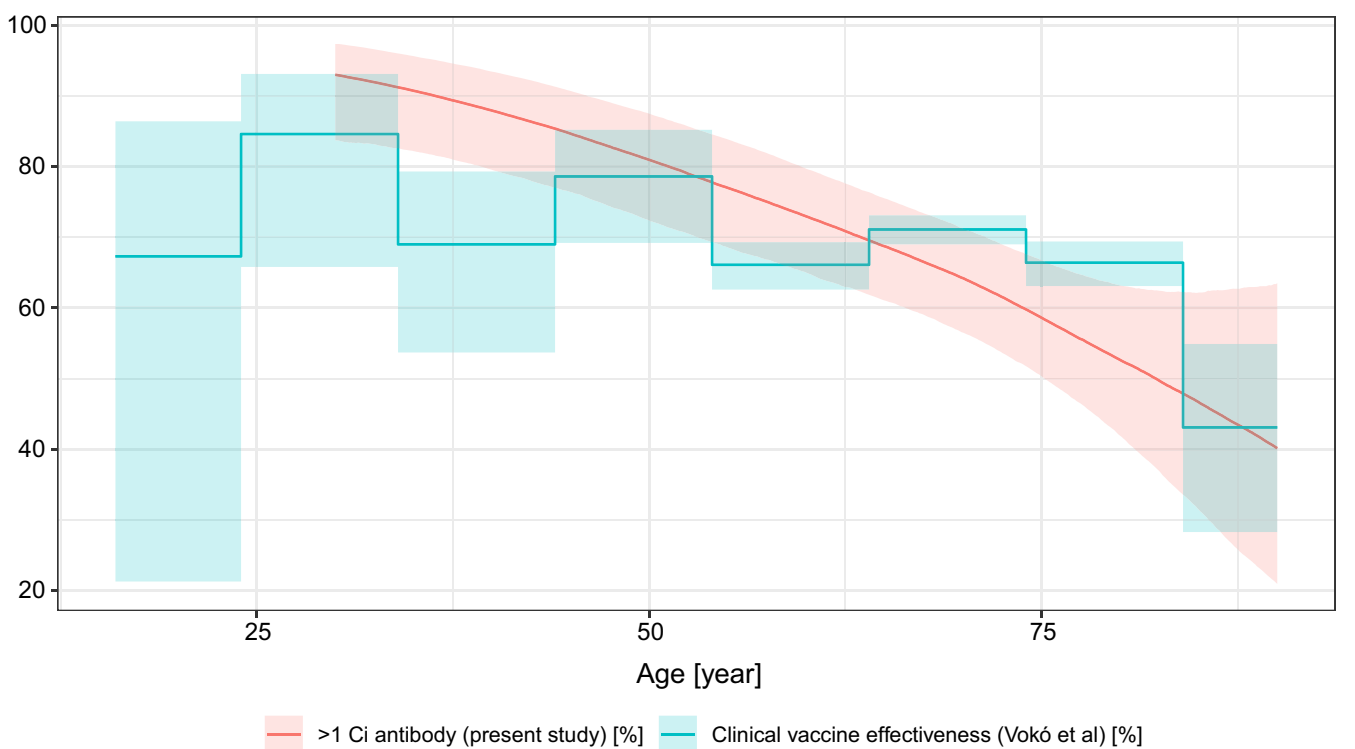

Fig. 8 Clinical vaccine effectiveness of the Sinopharm vaccine against infection from the Vokó et al. paper, overplotted with the predicted probability of antibody positivity (> 1 Ci titre) of the present study. Predictions were calculated for males (for visual clarity, as females would have been very similar, evidenced by Fig. 5) and 28 days after vaccination

of the COVID-19 disease, hospitalization or death and the type of vaccine administered, while being the most relevant evidence, may be difficult, especially in lowincome countries, and does not deliver individual-level information on susceptibility. However, it is relatively straightforward to analyze already available data on the RBD-specific antibody titres in the sera of subjects, which, when done with appropriate statistical tools and uniform laboratory methodology, can rapidly generate much-needed evidence assuming its good surrogacy.

The major strength of the present study is that it uses a relatively large sample (almost 450 subjects) for the Sinopharm vaccine with a uniform laboratory method and information on age, sex and time since vaccination, entered into a sophisticated statistical model. The sample size allowed the fine exploration of the effect of age (i.e., no categorization was used). To our best knowledge no such study has been published in the scientific literature, despite the fact that data on the protection conferred by the Sinopharm vaccine, and its age-dependence would be crucial for the more than 50 countries where this vaccine is authorized [29].

The study also has several limitations. First, the sample is not randomly taken from the population (to the contrary, it is likely that participants had a socioeconomic state above average for instance). This, however, is unlikely to have a major impact on antibody response, and especially on its age dependence. Also, while we excluded subjects with known previous SARS$\mathrm{CoV}-2$ virus infection, asymptomatic infection before vaccination cannot be ruled out. Note that this biases the probability estimate of having low titre after vaccination downwards, so the actual results can be even worse. Third, we had no information on the comorbidities of the subjects, as the study was primarily aimed at assessing the impact of age. Age is correlated with the presence of many chronic diseases, but the effect of these could not be separated in this study. The presented methodology however can be used to rapidly generate evidence in such groups, i.e., subjects with certain comorbidities too. Finally, while accumulating evidence points to the good surrogacy of the antibody titre used in the study, it is nevertheless not a direct measurement of disease susceptibility.

Based on the results presented here, we found that RBD-specific antibodies were present in a large percentage (about $90 \%$ ) of individuals below the age of 50 years, while this antibody production after Sinopharm vaccination was much less pronounced with increasing age. In fact, above the age of 60 , numerous subjects were found not to produce any protective antibody, reaching a percentage of up to $50 \%$ at older ages. When compared to our limited data set of the Pfizer/BioNTech vaccines (the results of which matched those found in major clinical studies [20]), the antibody titres after Sinopharm vaccination were almost an order of magnitude lower than after the Pfizer/BioNTech vaccine; in this latter case we did not find any individuals with no protective antibody. 


\section{Conclusion}

These findings call for the further evaluation of the protective antibody titres in the large population of elderly people vaccinated with the BBIBP-CorV (Sinopharm, Beijing CNBG) vaccine, and/or to provide additional vaccination of the potentially unprotected individuals. A significant danger is that if the lack of antibody production indeed translates to a weaker protection against the disease, while the Sinopharm vaccinated elderly feel and are declared to be protected against COVID-19, then this false promise may contribute to an outbreak of the disease in this highly vulnerable population.

\author{
Abbreviations \\ Ci Centaur index \\ COVID-19 Coronavirus disease 2019 \\ SARS-CoV-2Severe acute respiratory syndrome coronavirus 2
}

\section{Supplementary Information}

The online version contains supplementary material available at https://doi. org/10.1186/s12879-022-07069-z.

Additional file 1: Figure S1. Effects of the age and the sex of the subject, and the time-period between the vaccination and the measurement on the probability of the lack of RBD-specific antibody production (titre below 1) after two doses of the Sinopharm vaccine using logistic regression model. $90 \%$ credible interval is shown for males, 28 days post second dose. Figure S2. Effects of the age and the sex of the subject on the probability of the lack of RBD-specific antibody production (titre below 1) after two doses of the Pfizer/BioNTech vaccine using logistic regression model. $90 \%$ credible interval is shown for males, 28 days post second dose. Figure S3. Sinopharm vaccine model, MCMC diagnostics: density plot for the hurdle-lognormal model. Figure S4. Sinopharm vaccine model, MCMC diagnostics: density plot for the logistic model. Figure S5. Sinopharm vac cine model, MCMC diagnostics: trace plot for the hurdle-lognormal model. Figure S6. Sinopharm vaccine model, MCMC diagnostics: trace plot for the logistic model. Figure S7. Sinopharm vaccine model, MCMC diagnostics: autocorrelation function for the hurdle-lognormal model. Figure S8. Sinopharm vaccine model, MCMC diagnostics: autocorrelation function for the logistic model. Figure S9. Sinopharm vaccine model, MCMC diagnostics: posterior predictive check for the hurdle-lognormal model. Figure S10. Sinopharm vaccine model, MCMC diagnostics: posterior predictive check for the logistic model. Figure S11. Pfizer/BioNTech vaccine model, MCMC diagnostics: density plot for the hurdle-lognormal model. Figure S12. Pfizer/BioNTech vaccine model, MCMC diagnostics: density plot for the logistic model. Figure S13. Pfizer/BioNTech vaccine model, MCMC diagnostics: trace plot for the hurdle-lognormal model. Figure S14. Pfizer/ BioNTech vaccine model, MCMC diagnostics: trace plot for the logistic model. Figure S15. Pfizer/BioNTech vaccine model, MCMC diagnostics: autocorrelation function for the hurdle-lognormal model. Figure S16. Pfizer/BioNTech vaccine model, MCMC diagnostics: autocorrelation function for the logistic model. Figure S17. Pfizer/BioNTech vaccine model, MCMC diagnostics: posterior predictive check for the hurdle-lognormal model. Figure S18. Pfizer/BioNTech vaccine model, MCMC diagnostics: posterior predictive check for the logistic model.

\section{Acknowledgements}

The authors would like to thank Júlia Singer for her instructive comments that greatly improved the manuscript.

\section{Author contributions}

TF carried out the statistical analysis, verified the underlying data, participated in the interpretation of the results and revised the manuscript. BS generated the research question, conceived the study, did the literature review, curated and verified the data, and wrote the manuscript. All authors attest they meet the ICMJE criteria for authorship. All authors read and approved the final manuscript.

\section{Funding}

Open access funding provided by ELKH Research Centre for Natural Sciences. This research did not receive any specific grant from funding agencies in the public, commercial, or not-for-profit sectors.

\section{Availability of data and materials}

The datasets generated and/or analysed during the current study (including de-identified individual patient level data that was openly available to the public, and the full analysis script) are available in the GitHub repository https://github.com/tamas-ferenci/SinopharmAntibodyResponse.

\section{Declarations}

\section{Ethics approval and consent to participate}

All subjects participated with full written informed consent in the original data collection. Present analysis uses their data which was openly available to the public before the initiation of the study. The protocol for measurements was approved by the Hungarian health authorities.

\section{Consent for publication}

Not applicable (manuscript does not contain any individual person's data in any form)

\section{Competing interests}

The authors declare that they have no competing interests.

Received: 9 August 2021 Accepted: 10 January 2022

Published online: 24 January 2022

\section{References}

1. Hungarian government bill 19/2021. (I. 28.), and the corresponding permission of the Hungarian FDA (OGYÉl/5142-2/2021).

2. National Institute of Pharmacy and Nutrition. Alkalmazás előírás, SARSCoV-2 vakcina (VeroCell), inaktivált. https://www.ogyei.gov.hu/dynamic/ sinopharm vakcina taj_szakembereknek.pdf Accessed 10 June 2021.

3. European Centre for Disease Prevention and Control. COVID-19 vaccine tracker. https://vaccinetracker.ecdc.europa.eu/public/extensions/COVID19/vaccine-tracker.html. Accessed 21 June 2021.

4. Plotkin SA. Vaccines: correlates of vaccine-induced immunity. Clin Infect Dis. 2008:47:401-9.

5. Mallapaty S. Scientists zero in on long-sought marker of COVID-vaccine efficacy. Nature. 2021. https://doi.org/10.1038/d41586-021-01372-6.

6. Lumley SF, O'Donnell D, Stoesser NE, Matthews PC, Howarth A, Hatch SB, et al. Antibody status and incidence of SARS-CoV-2 infection in health care workers. N Engl J Med. 2021;384:533-40.

7. Harvey RA, Rassen JA, Kabelac CA, Turenne W, Leonard S, Klesh R, et al. Association of SARS-CoV-2 seropositive antibody test with risk of future infection. JAMA Intern Med. 2021:181:672.

8. Earle KA, Ambrosino DM, Fiore-Gartland A, Goldblatt D, Gilbert PB, Siber GR, et al. Evidence for antibody as a protective correlate for COVID-19 vaccines. Vaccine. 2021. https://doi.org/10.1016/j.vaccine.2021.05.063.

9. Addetia A, Crawford KHD, Dingens A, Zhu H, Roychoudhury P, Huang M-L, et al. Neutralizing antibodies correlate with protection from SARS-CoV-2 in humans during a fishery vessel outbreak with a high attack rate. J Clin Microbiol. 2020. https://doi.org/10.1128/JCM.02107-20.

10. Khoury DS, Cromer D, Reynaldi A, Schlub TE, Wheatley AK, Juno JA, et al. Neutralizing antibody levels are highly predictive of immune protection from symptomatic SARS-CoV-2 infection. Nat Med. 2021. https://doi.org/ 10.1038/s41591-021-01377-8 
11. Hall VJ, Foulkes S, Charlett A, Atti A, Monk EJM, Simmons R, et al. SARSCoV-2 infection rates of antibody-positive compared with antibodynegative health-care workers in England: a large, multicentre, prospective cohort study (SIREN). Lancet. 2021;397:1459-69.

12. Feng S, Phillips DJ, White T, Sayal H, Aley PK, Bibi S, et al. Correlates of protection against symptomatic and asymptomatic SARS-CoV-2 infection. Nat Med. 2021:27(11):2032-40

13. Letizia AG, Ge Y, Vangeti S, Goforth C, Weir DL, Kuzmina NA, et al. SARSCoV-2 seropositivity and subsequent infection risk in healthy young adults: a prospective cohort study. Lancet Respir Med. 2021;9:712-20.

14. Kool M, Fierens K, Lambrecht BN. Alum adjuvant: some of the tricks of the oldest adjuvant. J Med Microbiol. 2012;61:927-34.

15. Bungener L, Geeraedts F, ter Veer W, Medema J, Wilschut J, Huckriede A. Alum boosts TH2-type antibody responses to whole-inactivated virus influenza vaccine in mice but does not confer superior protection. Vaccine. 2008;26:2350-9.

16. Zhang Y, Zeng G, Pan H, Li C, Hu Y, Chu K, et al. Safety, tolerability, and immunogenicity of an inactivated SARS-CoV-2 vaccine in healthy adults aged $18-59$ years: a randomised, double-blind, placebo-controlled, phase 1/2 clinical trial. Lancet Infect Dis. 2021;21:181-92.

17. Xia S, Zhang Y, Wang Y, Wang H, Yang Y, Gao GF, et al. Safety and immunogenicity of an inactivated SARS-CoV-2 vaccine, BBIBP-CorV: a randomised, double-blind, placebo-controlled, phase 1/2 trial. Lancet Infect Dis. 2021;21:39-51.

18. Al Kaabi N, Zhang Y, Xia S, Yang Y, Al Qahtani MM, Abdulrazzaq N, et al. Effect of 2 inactivated SARS-CoV-2 vaccines on symptomatic COVID-19 infection in adults: a randomized clinical trial. JAMA. 2021. https://doi. org/10.1001/jama.2021.8565.

19. Choi WS, Cheong HJ. COVID-19 vaccination for people with comorbidities. Infect Chemother. 2021;53:155.

20. Polack FP, Thomas SJ, Kitchin N, Absalon J, Gurtman A, Lockhart S, et al. Safety and efficacy of the BNT162b2 mRNA Covid-19 vaccine. N Engl J Med. 2020;383:2603-15.

21. US Food and Drug Administration. Vaccines and related biological products advisory committee meeting December 10, 2020. FDA briefing document. Pfizer-BioNTech COVID-19 vaccine. https://www.fda. gov/media/144245/download. Accessed 27 June 2021.

22. Lord JM. The effect of ageing of the immune system on vaccination responses. Hum Vaccines Immunother. 2013;9:1364-7.

23. Van Der Meeren O, Crasta P, Cheuvart B, De Ridder M. Characterization of an age-response relationship to GSK's recombinant hepatitis B vaccine in healthy adults: an integrated analysis. Hum Vaccines Immunother. 2015;11:1726-9.

24. McLean HQ, Thompson MG, Sundaram ME, Kieke BA, Gaglani M, Murthy $K$, et al. Influenza vaccine effectiveness in the United States during 2012-2013: variable protection by age and virus type. J Infect Dis. 2015;211:1529-40.

25. Collier DA, Ferreira IATM, Kotagiri P, Datir RP, Lim EY, Touizer E, et al. Age-related immune response heterogeneity to SARS-CoV-2 vaccine BNT162b2. Nature. 2021;596:417-22.

26. Stark K, Günther M, Neuhaus R, Reinke P, Schröder K, Linnig S, et al. Immunogenicity and safety of hepatitis A vaccine in liver and renal transplant recipients. J Infect Dis. 1999;180:2014-7.

27. Bosaeed M, Kumar D. Seasonal influenza vaccine in immunocompromised persons. Hum Vaccines Immunother. 2018;14:1311-22.

28. Rabinowich L, Grupper A, Baruch R, Ben-Yehoyada M, Halperin T, Turner $D$, et al. Low immunogenicity to SARS-CoV-2 vaccination among liver transplant recipients. J Hepatol. 2021;75:435-8.

29. McGill COVID19 Vaccine Tracker Team. COVID19 vaccine tracker: Sinopharm (Beijing): BBIBP-CorV. https://covid19.trackvaccines.org/vacci nes/5/. Accessed 28 June 2021.

30. World Health Organization. WHO lists additional COVID-19 vaccine for emergency use and issues interim policy recommendations. https:// www.who.int/news/item/07-05-2021-who-lists-additional-covid-19vaccine-for-emergency-use-and-issues-interim-policy-recommenda tions. Accessed 7 May 2021

31. Ocmant A, Roisin S, De Meuter R, Brauner J. Clinical performance of the Advia Centaur anti-SARS-CoV-2 chemiluminescent immunoassay related to antibody kinetics. J Med Virol. 2021;93:2583-4.
32. Müller L, Ostermann PN, Walker A, Wienemann T, Mertens A, Adams O, et al. Sensitivity of anti-SARS-CoV-2 serological assays in a high-prevalence setting. Eur J Clin Microbiol Infect Dis. 2021;40:1063-71.

33. Shi AC, Ren P. SARS-CoV-2 serology testing: progress and challenges. J Immunol Methods. 2021:494:113060.

34. Centers for Disease Control and Prevention (CDC). Interim guidelines for COVID-19 antibody testing in clinical and public health settings updated Mar. 17, 2021. https://www.cdc.gov/coronavirus/2019-ncov/lab/resou rces/antibody-tests-guidelines.html.

35. Centers for Disease Control and Prevention (CDC). Coronavirus disease 2019: evaluation and testing. https://www.cdc.gov/coronavirus/2019nCoV/hcp/clinical-criteria.html.

36. World Health Organization. Coronavirus disease (COVID-19) technical guidance: laboratory testing for 2019-nCoV in humans page. https:// www.who.int/emergencies/diseases/novel-coronavirus-2019/technicalguidance/laboratory-guidance.

37. Mulligan MJ, Lyke KE, Kitchin N, Absalon J, Gurtman A, Lockhart S, et al. Phase I/II study of COVID-19 RNA vaccine BNT162b1 in adults. Nature. 2020;586:589-93.

38. Chen X, Li R, Pan Z, Qian C, Yang Y, You R, et al. Human monoclonal antibodies block the binding of SARS-CoV-2 spike protein to angiotensin converting enzyme 2 receptor. Cell Mol Immunol. 2020;17:647-9.

39. Huang Y, Sun H, Yu H, Li S, Zheng Q, Xia N. Neutralizing antibodies against SARS-CoV-2: current understanding, challenge and perspective. Antib Ther. 2020;3:285-99.

40. Saunders KO, Lee E, Parks R, Martinez DR, Li D, Chen H, et al. Neutralizing antibody vaccine for pandemic and pre-emergent coronaviruses. Nature. 2021;594:553-9.

41. Wood SN. Thin plate regression splines: thin plate regression splines. J R Stat Soc Ser B Stat Methodol. 2003;65:95-114.

42. R Core Team. R: a language and environment for statistical computing. R Foundation for Statistical Computing, Vienna, Austria. 2021. https:// www.R-project.org/.

43. Bürkner P-C. brms: an $R$ package for Bayesian multilevel models using Stan. J Stat Softw. 2017. https://doi.org/10.18637/jss.v080.i01.

44. Feng Y, Chen J, Yao T, Chang Y, Li X, Xing R, et al. Safety and immunogenicity of inactivated SARS-CoV-2 vaccine in high-risk occupational population: a randomized, parallel, controlled clinical trial. Infect Dis Poverty. 2021;10(1):138.

45. Ariamanesh M, Porouhan P, PeyroShabany B, Fazilat-Panah D, Dehghani $M$, Nabavifard $M$, et al. Immunogenicity and safety of the inactivated SARS-CoV-2 vaccine (BBIBP-CorV) in patients with malignancy. Cancer Invest. 2021;40(1):26-34.

46. Alqassieh R, Suleiman A, Abu-Halaweh S, Santarisi A, Shatnawi O, Shdaifat $L$, et al. Pfizer-BioNTech and Sinopharm: a comparative study on postvaccination antibody titers. Vaccines. 2021;9:1223.

47. Fu Y, Chen F, Cui L, Zhao Y, Zhang H, Fu S, et al. Immunological analysis of people in Northeast China after SARS-CoV-2 inactivated vaccine injection. Vaccines. 2021;9:1028.

48. Vályi-Nagy I, Matula Z, Gönczi M, Tasnády S, Bekő G, Réti M, et al. Comparison of antibody and T cell responses elicited by BBIBP-CorV (Sinopharm) and BNT162b2 (Pfizer-BioNTech) vaccines against SARS-CoV-2 in healthy adult humans. GeroScience. 2021:43:2321-31.

49. Lijeskić O, Klun I, Stamenov Djaković M, Gligorić N, Štajner T, Srbljanović $J$, et al. Prospective cohort study of the kinetics of specific antibodies to SARS-CoV-2 infection and to four SARS-CoV-2 vaccines available in Serbia, and vaccine effectiveness: a 3-month interim report. Vaccines. 2021:9:1031.

50. Dashdorj NJ, Wirz OF, Röltgen K, Haraguchi E, Buzzanco AS, Sibai M, et al. Direct comparison of antibody responses to four SARS-CoV-2 vaccines in Mongolia. Cell Host Microbe. 2021;29(12):1738-43.

51. Jeewandara C, Aberathna IS, Pushpakumara PD, Kamaladasa A, Guruge D, Jayathilaka D, et al. Antibody and T cell responses to Sinopharm/BBIBPCorV in naïve and previously infected individuals in Sri Lanka. Preprint, medRxiv. 2021. https://doi.org/10.1101/2021.07.15.21260621.

52. Jeewandara C, Aberathna IS, Pushpakumara PD, Kamaladasa A, Guruge $D$, Wijesinghe A, et al. Persistence of antibody and T cell responses to the Sinopharm/BBIBP-CorV vaccine in Sri Lankan individuals. Preprint, medRxiv. 2021. https://doi.org/10.1101/2021.10.14.21265030.

53. Li X-N, Huang Y, Wang W, Jing Q-L, Zhang C-H, Qin P-Z, et al. Effectiveness of inactivated SARS-CoV-2 vaccines against the Delta variant infection 
in Guangzhou: a test-negative case-control real-world study. Emerg Microbes Infect. 2021;10:1751-9.

54. Macchia A, Ferrante D, Angeleri P, Biscayart C, Mariani J, Esteban S, et al. Evaluation of a COVID-19 vaccine campaign and SARS-CoV-2 infection and mortality among adults aged 60 years and older in a middle-income country. JAMA Netw Open. 2021;4:e2130800.

55. Vokó Z, Kiss Z, Surján G, Surján O, Barcza Z, Pályi B, et al. Nationwide effectiveness of five SARS-CoV-2 vaccines in Hungary-the HUN-VE study. Clin Microbiol Infect. 2021. https://doi.org/10.1016/j.cmi.2021.11.011.

\section{Publisher's Note}

Springer Nature remains neutral with regard to jurisdictional claims in published maps and institutional affiliations.

- fast, convenient online submission

- thorough peer review by experienced researchers in your field

- rapid publication on acceptance

- support for research data, including large and complex data types

- gold Open Access which fosters wider collaboration and increased citations

- maximum visibility for your research: over $100 \mathrm{M}$ website views per year

At $\mathrm{BMC}$, research is always in progress.

Learn more biomedcentral.com/submissions 